\title{
HACIA UNA NORMA ESPECÍFICA CONTABLE AMBIENTAL: RECONOCIMIENTO, MEDICIÓN E INFORMACIÓN INTEGRAL
}

\author{
TOWARDS A STANDARD SPECIFIC ENVIRONMENTAL ACCOUNTING: \\ RECOGNITION AND COMPREHENSIVE MEASUREMENT INFORMATION \\ Elsa Esther Choy Zevallos* \\ Docente Asociada de la Facultad de Ciencias Contables \\ Universidad Nacional Mayor de San Marcos-UNMSM / Lima-Perú \\ [Recepción: Marzo de 2014/ Conformidad: Mayo 2014]
}

\section{RESUMEN}

La problemática del impacto ambiental en el mundo, permite admitir la presencia del surgimiento de una nueva disciplina contable de información económico-financiero, con cambios en el marco conceptual y en las normas específicas que agreguen valor a la contabilidad tradicional. Esto implicaría la adopción de nuevos métodos de medición, estimaciones y actualización conceptual sobre activos, pasivos, gastos, costos y contingencias; con las particularidades que rodea al "medio ambiente", llevándolo a un análisis más cualitativo que cuantitativo. Un reto para la ciencia contable e injerencia de otras ciencias, que provean mayor conocimiento.

No existe una norma específica contable ambiental ante este vacío, se están utilizando ciertas normas vigentes que plantean un tratamiento genérico para situaciones ambientales como: NIC 16, NIC 36, NIC 37 y NIC 38.

El objetivo es expresar la necesidad de establecer los fundamentos para un marco conceptual y el alcance de la norma ambiental, bases para su reconocimiento, medición y exposición, a fin de lograr información contable integral.

Actualmente, el tema se encuentra en proceso de investigación, que tiene un enfoque metodológico deductivo de tipo descriptivo. Al final, se pretende presentar una estructura de los fundamentos para un marco conceptual ambiental.

\section{Palabras Clave:}

Norma contable ambiental; marco conceptual; reconocimiento medición; contabilidad tradicional.

\begin{abstract}
The issue of environmental impact in the world, let's admit the presence of the emergence of a new accounting business and financial discipline, with changes in the conceptual framework and the specific rules that add value to traditional accounting information. This would involve the adoption of new measurement methods, estimates and conceptual update on assets, liabilities, expenses, costs and liabilities; with the particulars surrounding the "environment", leading him to a more qualitative than quantitative analysis. A challenge for the accounting science and interference from other sciences, to provide more knowledge. There is no specific environmental accounting standard before this vacuum, using certain existing rules are presenting a generic treatment for environmental situations such as: NIC 16, NIC 36, NIC 37 and NIC 38. The goal is to express the need to establish the foundation for a conceptual framework and scope of the environmental standard, bases for recognition, measurement and disclosure, in order to achieve comprehensive accounting information. Currently, the issue is under investigation, which has a deductive descriptive methodological approach. In the end, we intend to present a structure of the foundations for an environmental framework.
\end{abstract}

\section{Keywords:}

Environmental accounting standard; conceptual framework; measurement recognition; traditional accounting.

\footnotetext{
* Doctora en Ciencias Contables y Empresariales, Magister en Dirección Financiera y Contador Público Colegiado.
} Email: eschoyz@outlook.com 


\section{INTRODUCCIÓN}

La inserción de la economía peruana en mercados internacionales y la firma de tratados de libre comercio, exigen al gobierno desempeñar un nuevo papel en el logro de un desarrollo sustentable para la formación de una nueva cultura ecológica, calidad de vida y conservación del entorno natural, la cual requiere de un trabajo articulado con las organizaciones de la sociedad civil y organismos profesionales. Con la implementación de una norma contable ambiental, se crea las condiciones para que los profesionales de las ciencias contables y otras disciplinas, inicien un proceso de concientización, revisión de su papel y de su acción para contribuir de manera más efectiva en la búsqueda de un desarrollo sustentable.

Este trabajo pretende señalar la necesidad de una metodología útil para el desarrollo de sistemas de información contable integrados que tiendan a satisfacer las necesidades de los distintos tipos de organizaciones. En este sentido, la información ambiental genera un valor agregado a la hora de tomar decisiones.

\section{TEORÍA CONTABLE Y ASPECTOS AMBIENTALES}

Con la finalidad de lograr un análisis comparativo de la teoría contable y la información ambiental, presentamos la investigación del doctor Carlos Luis García Casella, Profesor Emérito de la Facultad de Ciencias Económicas de la Universidad de Buenos Aires. Su trabajo fundamenta la contabilidad como una ciencia empírica, factual y aplicada que no solo se ocupa de los fenómenos económicos. Se sustenta en el pensamiento de Richard Mattessich pero corrigiendo el carácter y sesgo economicista y empresarial. Si bien, la teoría general contable de Mattessich pretende ser universal y polivalente, la descripción y la definición de los supuestos, axiomas y teoremas reducen el saber contable; García Casella percibe esta situación, lo que le permite reformular estas estructuras conceptuales tendiendo a una concepción más universal y amplia de la contabilidad.

Desde la concepción del mencionado profesor, la contabilidad puede ser patrimonial, de gestión, gubernamental, económica y social. El mismo autor presenta una serie de "hipótesis descartadas" que hacen referencia a consideraciones reduccionistas y parciales de la contabilidad. A continuación, se presenta algunas de las "hipótesis descartadas" relacionadas con acciones ambientales. (García 2006, pp.14 - 5).

- Es falso que la contabilidad se ocupa "solamente de fenómenos económicos". La hipótesis se descarta porque los aspectos económicos son parte de los temas objeto de estudio de la contabilidad pero su alcance cubre aspectos sociales, ambientales, culturales y otros, que son susceptibles de ser informados y que representen interés para usuarios determinados.

- La contabilidad no puede tratar "solamente cuestiones patrimoniales", este aspecto es solo un segmento de los distintos tópicos necesarios para ofrecer una información acorde a las diferentes necesidades de los usuarios.

- Cuando se presenta solamente información patrimonial, se desconoce la necesidad de informes en materia de gestión, gubernamental, social, ambiental y económica.

- La orientación que considera que "la contabilidad es la ciencia del control económico” desconoce los otros campos donde la contabilidad actúa; entre ellos, ambiental, social, gubernamental, gerencial $y$ financiero.

- Frente a la anterior hipótesis descartada, el autor propone que "la contabilidad abarca tareas de control económico como una pequeña parte del dominio o universo del discurso contable".

- La contabilidad es cualitativa y cuantitativa; expresa aspectos en términos monetarios, pero existen realidades que son objeto de la contabilidad que no se expresan monetariamente, de forma que no se puede decir que "la Contabilidad es exclusivamente monetaria”.

El profesor García, ampliando tal reduccionismo, manifiesta que "no es posible expresar las relaciones y mediciones contables exclusivamente en términos monetarios". El modelo contable financiero ha utilizado históricamente la moneda como unidad de expresión; los modelos contables sociales y ambientales requieren la utilización de unidades de medida físicas pues la moneda no es un instrumento ade- 
cuado para la representación de segmentos sociales y ambientales.

\section{NORMAS INTERNACIONALES DE CONTABILIDAD}

Actualmente, no existe una norma especial para acciones ambientales, algunas de las Normas Internacionales de Contabilidad que se encuentran aún vigentes, señalan en forma particular y específica, el tratamiento contable que deben recibir las acciones ambientales realizadas por las entidades y se limitan exclusivamente a realizar referencias indirectas ante la presencia de gastos o pasivos ambientales; reales o contingentes, como la NIC 36 Deterioro del Valor de los Activos, la NIC 37 Activos Contingentes, Pasivos Contingentes y Previsiones y, la NIC 38 Intangibles, en las cuales el concepto de gasto y obligaciones medioambientales están incluidas algunas veces, en forma indirecta junto a los demás gastos y obligaciones, recibiendo el mismo tratamiento que las otras partidas.

En nuestro país, la incorporación de un activo de larga duración al patrimonio de un ente, así como los progresos sobre los mismos, se encuentran regulados por las normas contables profesionales, tales como la NIC 16 Propiedades, Planta y Equipo, el concepto de activo incorpora en su definición beneficios económicos futuros y su costo medido con fiabilidad. Sin embargo, para el logro de la sustentabilidad ambiental, se requiere la incorporación de bienes o la modificación de los mismos no necesariamente relacionada a la generación de dichos beneficios o al menos no relacionada de manera directa, como parecería ceñirse la definición de la norma respecto a los costos iniciales. La NIC 16 (párrafo 11) establece que algunos elementos de propiedades, planta y equipo pueden ser adquiridos por razones de seguridad o de índole medioambiental. Sin embargo, aunque la adquisición de ese tipo de propiedades, planta y equipo no incremente los beneficios económicos que proporcionan las partidas existentes, puede ser necesaria para que la entidad logre obtener los beneficios económicos derivados del resto de los activos. Las obligaciones por los costos contabilizados según la NIC 16 se reconocerán y medirán de acuerdo con la
NIC 37 Provisiones, Pasivos Contingentes y Activos Contingentes.

Por otro lado, la unidad monetaria no refleja realmente la situación del medio ambiente, pero si refleja la situación financiera de la economía. Por lo tanto, es necesario buscar un mecanismo que permita hacer un acercamiento en términos de unidades de medida diferentes para reconocer esta situación.

La ONU plantea a este problema una solución a nivel de la contabilidad nacional, Cuentas Nacionales, y lo plasma en el manual llamado Sistema de Contabilidad Ambiental y Económica Integrada, que plantea una estructura de cuentas satélites compuestas por matrices macroeconómicas, donde se refleja la información de la relación entre la economía y el medio ambiente en unidades como volumen, energía, etc. yluego, la compara con la información macroeconómica recogida en unidades monetarias.

Por último, la normativa contable emitida por International Accounting Standards Board (IASB) de las cuestiones ambientales, recomienda la inclusión de pasivos y activos ambientales en la información financiera de las empresas, así como también, de contingencias y costos referidos al medio ambiente.

\section{FUNDAMENTO METODOLÓGICO PARA UN MARCO CONCEPTUAL}

Según la Asociación Española de Contabilidad y Administración de Empresas (AECA), "la contabilidad ambiental es una rama de la contabilidad de muy reciente reconocimiento y, quizás por ello, es frecuente encontrar términos comunes con significados diferentes, cuando no contradictorios".

Surge así, la necesidad de establecer unos fundamentos metodológicos para construir el marco conceptual y, por lo tanto, el alcance de la contabilidad ambiental, siendo éste el propósito de este trabajo.

La AECA señala que la contabilidad ambiental puede definirse como la parte de la contabilidad aplicada cuyo objeto son las relaciones entre una entidad y su medio ambiente, lo cual supone diferenciar entre la macro y la micro contabilidad y, dentro de ésta última, precisar tanto el concepto de entidad como el de medio ambiente. 
Cuando la delimitación de la entidad rige a partir de criterios jurídicos y el medio ambiente como entorno natural, el marco conceptual de la norma ambiental se fundamenta en la economía tradicional, para lo cual, la ciencia económica es independiente de cualquier consideración ambiental, excepto en dos aspectos: la naturaleza como productora de recursos económicos, susceptibles de cuantificarse y transformarse y, como receptora de desechos. Es así, que un marco conceptual ambiental debe proporcionar información que atienda al principio clásico de entidad y se reduzca a los inputs adicionales necesarios para prevenir, reducir y eliminar la contaminación y a los outputs no deseados como residuos, vertidos, emisiones. Esta es la posición adoptada en las normas de contabilidad financiera emitidas por el IASC (1998), la Unión Europea (2001b) y el Instituto de Contabilidad y Auditoría de cuentas (2002).

Si el criterio se deriva del análisis del ciclo de vida de sus productos y actividades y el medio ambiente se entiende como entorno vital, el marco conceptual se fundamenta en la economía ecológica, para lo cual el sistema económico es un subsistema del sistema ambiental, acorde con sus normas y en continua interacción con él. En este caso, el marco conceptual ambiental precisa de un nuevo principio de entidad, a fin de ofrecer información acerca de todo tipo de inputs y outputs sociales. Esta es la posición más generalizada entre aquellos que promueven el desarrollo de la contabilidad ambiental en el ámbito de la responsabilidad social, como el Global Reporting Initiative GRI (2002) o la Comisión de Responsabilidad Social Corporativa de AECA.

Otro criterio en el cual el marco conceptual se fundamenta en la economía ambiental, posición intermedia entre la economía tradicional y la economía ecológica, donde el sistema económico está relacionado y limitado por el sistema ambiental, planteadas por las normas ISO de las series 14000 y 19000.

Por último, el criterio, en la cual el marco conceptual ha de estar dotado de suficiente flexibilidad para permitir la adaptación a los objetivos previamente fijados, de la información contable demandada. Este planteamiento propio de la contabilidad de gestión, permite combinar los diversos criterios de delimitación de la entidad y del medio ambiente, aplicándolos en función de los objetivos que persiga la información contable. En este caso, el marco conceptual se fundamenta en la economía ambiental, posición intermedia entre la economía tradicional y la economía ecológica, para lo cual el sistema económico está relacionado y limitado por el sistema ambiental. Esta alternativa subyace en el documento 13 de la Comisión de Contabilidad de Gestión de la Asociación Española de Contabilidad y Administración de Empresas-AECA (1996) en las normas ISO de las series 14000 (1996) y 19000 (2003), el reglamento EMAS de la Unión Europea (2001a).

La ampliación de la información contable al ámbito ambiental demanda fundamentos metodológicos y lenguaje común e interdisciplinario.

\section{HACIA UN MARCO CONCEPTUAL AMBIENTAL}

Complementando lo antes expuesto, consideramos adecuado construir un marco conceptual ambiental, tomando como base el marco conceptual para la presentación y exposición de la información financiera. En tal sentido, los temas centrales corresponden a:

a) Objetivos de la información medioambiental.

b) Alcance de la información medioambiental.

c) Partes que compone el informe medioambiental.

d) Características cualitativas de la información.

e) Definición de los elementos que componen la información.

f) Reconocimiento e información de cada uno de los elementos.

g) Medición de los elementos.

Existe una propuesta de estructura conceptual ambiental que la consideramos adecuada para desarrollar un marco conceptual. 
HACIA UNA NORMA ESPECÍFICA CONTABLE AMBIENTAL: RECONOCIMIENTO, MEDICIÓN E INFORMACIÓN INTEGRAL

Modelo: Estructura lineamientos para un Marco Conceptual Ambiental

\begin{tabular}{|c|c|}
\hline \multicolumn{2}{|c|}{ Marco relativo del sistema de información contable } \\
\hline \multicolumn{2}{|c|}{ Marco de compromiso y objetivos } \\
\hline Responsabilidad & Objetivos \\
\hline \multicolumn{2}{|c|}{ Marco conceptual } \\
\hline \multirow{2}{*}{ Conceptos cualitativos } & Conceptos cuantitativos \\
\hline & Elementos \\
\hline $\begin{array}{l}\text { - Relevancia } \\
\text { - Valor de predicción } \\
\text {-Valor de realimentación } \\
\text { - Valor de exactitud } \\
\text { - Confiabilidad } \\
\text { - Verificable } \\
\text { - Presentación fidedigna } \\
\text { - Neutralidad } \\
\text { - Consistencia }\end{array}$ & $\begin{array}{l}\text { - Información presupuestaria ambiental } \\
\text { - Ingresos ambientales } \\
\text { - Inversión y desarrollo ambiental } \\
\text { - Compras y consumo de recursos naturales } \\
\text { - Materiales energéticos } \\
\text { - Reutilizados, reciclados y valorizados } \\
\text { - Personal } \\
\text { - Efectos no deseados } \\
\text { - Información financiera ambiental } \\
\text { - Activos ambientales } \\
\text { - Contingencias ambientales } \\
\text { - Provisiones ambientales } \\
\text { - Ingresos ambientales } \\
\text { - Gastos ambientales } \\
\text { - Coste ambiental }\end{array}$ \\
\hline
\end{tabular}

\begin{tabular}{|l|l|l|}
\hline \multicolumn{2}{|c|}{ Conceptos de reconocimiento y medida } \\
\hline Afirmaciones básicas & Criterios valorativos & \multicolumn{1}{c|}{ Limitaciones } \\
\hline - Entidad económica y me- & - Costo histórico & - Importancia relativa o mate- \\
dio ambiental & - Valor razonable & rialidad. \\
- Entidad en marcha & - Devengado & - Práctica conservadora \\
- Unidad de medida & - Correlación de ingresos y & - Costo-beneficio de informa- \\
- Periodo contable & gastos & ción \\
& & - Práctica de otros modelos \\
\hline
\end{tabular}

\begin{tabular}{|l|l|}
\hline \multicolumn{2}{|c|}{ Desempeño ambiental } \\
\hline \multicolumn{2}{|c|}{ Razones o indicadores } \\
\hline Financieros & No Financieros \\
\hline- Gasto ambiental & - Agua \\
- Costos ambientales & - Energía \\
- Pérdidas ambientales & - Materiales \\
- Inversiones ambientales & - Emisiones, vertidos y residuos \\
- Activos ambientales & - Productos y servicio \\
- Ingresos ambientales & \\
\hline
\end{tabular}

Autor: Marino José Palacios Copete

Docente investigador de la Facultad de Administración de Empresas y Contabilidad de la Universidad de Panamá. 


\section{CONCLUSIONES}

1. El nuevo enfoque de la teoría contable permite afirmar que la contabilidad se ocupa de la medición en un sentido amplio, abarcando la dimensión cualitativa y la cuantitativa, no sólo se limita a las mediciones monetarias, también utiliza diversas escalas y unidades de medida.

2. La información contable ambiental abarca una dimensión más cualitativa que cuantitativa, por tanto la exactitud y la precisión de las mediciones son relativas. Se evidencia la necesidad e importancia de establecer los fundamentos teóricos para elaborar un marco conceptual en situaciones ambientales, de modo que permita la práctica de políticas contables efectivas y económicamente eficientes, para un manejo sustentable del medio ambiente.

3. El marco conceptual ambiental permite a la empresa contar con información para conocer cuál es su desempeño ambiental, desarrollar estrategias corporativas en el marco de su responsabilidad social con el medio ambiente, con la finalidad de disminuir los aspectos ambientales, reducir costes, mejorar la eficiencia y tener una ventaja competitiva.

4. La complejidad que presenta la información ambiental hace necesaria la participación de los profesionales de las distintas disciplinas, a fin establecer los lineamientos y métodos de reconocimiento y, medición de bienes y servicios ambientales.

\section{RECOMENDACIONES}

1. El gobierno del Perú debe establecer políticas efectivas y eficientes en asuntos ambientales, debido a la inserción de la economía peruana en mercados internacionales y la firma de tratados de libre comercio.

2. Es necesario que los empresarios e industriales tomen conciencia de aplicar políticas ambientales en los procesos de producción, a fin de conservar el medio ambiente que les rodea y permita un manejo sustentable del inventario de activos y recursos ambientales renovables y no renovables.
3. Corresponde al Ministerio del Ambiente identificar y valorar desde un punto de vista cuantitativo y cualitativo, los espacios naturales en nuestro país, a fin de tomar decisiones estratégicas para el aprovechamiento de los recursos naturales, así como, la credibilidad y legitimidad de la autoridad ambiental al momento de sancionar y castigar a los agentes que dañan o no cumplen con las normas, destinando lo recaudado a remediar el daño ambiental.

4. Es necesario la participación de los profesionales de las distintas disciplinas en el desarrollo de un marco conceptual ambiental, que sirva como base hacia un futuro previsible y progresivo de una norma contable específica para acciones ambientales.

\section{REFERENCIAS BIBLIOGRÁFICAS}

1. Asociación Española de Contabilidad y Administración de Empresa (AECA), (2003). Marco conceptual de la responsabilidad social Corporativa. Documento No.1.

2. Asociación Española de Normalización y Certificación (AENOR), (2005). Gestión ambiental.

3. Blanco, R.E. R. (2006). Influencia de la legislación en la información medioambiental suministrada por las empresas. Tesis Doctoral. Universidad Rey Juan Carlos. 329 Páginas.

4. García Casella Carlos Luis (2006). Modelización posible de la contabilidad social, 15-22. Buenos Aires: Ediciones Cooperativas.

5. Cañibano, C. L. y Gonzalo, A. J. A. (1995). Los programas de investigación en contabilidad, $1^{\text {a }}$ Jornada sobre teoría de la contabilidad.

6. Fernández, C. C. (2006). La responsabilidad social y medio ambiental: nuevos rumbos para la contabilidad. Revista Contabilidad y Auditoría, V. XII N. 24 P. 15-28, Argentina.

7. Fronti de García, Luisa y García Casella, Carlos Luis (2009). El sistema contable de gestión ambiental ante el cambio climático.Buenos Aires: Universidad de Buenos Aires.

8. Global Reporting Initiative (GRI) (2006). Protocolo de los indicadores G3: Medio Ambiente. 
9. Gómez, L. R. (2002). Ciencia Contable: Fundamentos científicos y metodológicos.

10. ISO/IEC 14001-2006 Sistema de gestión ambiental. 33 Páginas.

11. Larrinaga, G. C. (1999). ¿Es la contabilidad Medioambiental un paso hacia La sostenibilidad o un escudo Contra el cambio? El caso del sector eléctrico español. Revista Española de Financiación y Contabilidad Vol. XXVLII, 11 ' 101 julio-septiembre pp 645-674.

12. Masanet, L. M.J. (2005). Desarrollo e Integración de los Sistemas de Información Contable en la Gestión Medioambiental de la Empresa, Tesis Doctoral, Universitat Jaume I, 379 páginas.
13. Montesinos, J.V. (1978). Formación histórica, corrientes doctrinales y programas de investigación de la Contabilidad. Revista Técnica Contable. Vol. XXX, No 351 a 358, marzo octubre, 74 Páginas.

14. Navarro, V. M., Rodrigo, M. y Fernández, C. (2005). De la teoría a la práctica: el pensamiento de Virginia Henderson en el siglo XXI. $3^{\text {a }}$ edición. Publicación Elsrvier Masson. 191 Páginas.

15. Organización de las Naciones Unidas (ONU), (1992). Declaración de Rio sobre el Medio Ambiente y el Desarrollo.

16. Palacios Copete, Marino José. Facultad de Administración de Empresas y Contabilidad Universidad de Panamá. 